\title{
Theoretical Investigations on CPW-Fed Single and Dual-Polarized Slot Radiators Using Schelkunoff's Biconical Antenna Analysis
}

\author{
Amartya BANERJEE, Kaushik PATRA, Sayan CHATTERJEE, \\ Bhaskar GUPTA, Anup Kumar BANDYOPADHYAY \\ Dept. of ETCE, Jadavpur University, 188 Raja S. C. Mallick Road, Kolkata - 700032, India \\ amartyapotter@gmail.com, kaushikpatra294@gmail.com, sayan1234@gmail.com, \\ gupta_bh@yahoo.com, akbandyopadhyay@gmail.com
}

Submitted August 2, 2019 / Accepted February 27, 2020

\begin{abstract}
This article presents a closed-form analysis of $C P W$-fed slot dipole structures with the help of Schelkunoff's biconical antenna analysis technique and Babinet's principle. Input characteristics of $C P W$-fed slot dipole antennas are investigated, and closed-form expressions are derived for the purpose. The feed-gap inherently generated in $C P W$-fed antenna configurations is accounted for in the expressions, and the analysis of Schelkunoff is modified to address the same. Single-polarized structures can be orthogonally placed to generate dual-polarized characteristics - this notion is utilized to extend the proposed structure of a single CPW-fed slot dipole radiator towards a dual-polarized configuration. The proposed theoretical expressions are further validated for the dualpolarized geometry, and good agreement is observed in concerned theoretical and measured results. The simplicity of the proposed expressions is evident as they entirely consist of Sine and Cosine integrals and facilitate faster computation. Schelkunoff's Biconical Antenna method is rarely used for solving a planar slot radiator problem which justifies the novelty of this article. The present work for the first time - modifies the method of Schelkunoff to further account for the inherently generated feed-gap in $C P W$-fed planar monopole or dipole configurations.
\end{abstract}

\section{Keywords}

CPW-fed slot radiator, dual-polarized slot radiator, input impedance investigation, Schelkunoff's Biconical Antenna analysis

\section{Introduction}

In a multiuser environment, for maintaining ubiquitous connectivity in a seamless manner, it is required to have omnidirectional transmission and reception capabilities for the antennas, maintaining high isolation between the employed channels. Traffic capacities are enhanced in such multi-user multi-channel systems by using diversely polarized antenna configurations [1]. Planar slot antennas have become suitable candidates for such communication systems owing to their ease of fabrication and attractive performances [2-6]. In this context, narrower slots offer less bandwidth but better polarization purity in case of practical usage [7]. Therefore, for polarization diversity applications - narrow-slot structures are preferred as they ensure adequate isolation between the ports [8]. Orthogonally placed narrow-slot antennas or feed structures are used to generate dual-polarized radiation as reported in [9], [10]. With the advent of computers and numerical techniques for electromagnetic problem solving, closed form analyses for planar antennas are less observed in recent times and empirical guidelines are mostly followed for designing purposes. The techniques presently used [11-13] for analyses are complex and they demand extensive resources in terms of cost and computation. It is therefore always worth to investigate the problems with an aim to develop a more straightforward method for analysis that will provide closed-form expressions.

In 1946, Booker [14] explained that a half-wave slot on a metal sheet could be viewed as a resonant antenna resembling a half-wave dipole - extending Babinet's principle of optics to electromagnetics. This opened up an opportunity to analyze new problems with the help of old solutions. To analyze printed slot antennas, expressions derived for printed strip dipole structures can be extended using the work of Booker [14] to map the same on their slotted counterparts. Printed strip antennas can be investigated by solving the current integral equation in spectral or space domain as suggested in [15], [16]. In [15] the method becomes complex if it takes the radiating space into account. The work in [16], which utilizes free-space Green's Function in the kernel of the integral equation, leads to the evaluation of a more complicated function owing to the unknown parameters of the strip and the dielectric. Based on space-domain integral equation solutions - some other 
researchers considered equivalent wire dipole structures to represent the strip radiators as reported in [17-19]. In [20], the authors extended the analysis for printed strip dipoles to analyze printed slots on dielectric - thus validating the fact that from cylindrical wire dipole antennas one can map the solutions to printed strips and their slotted counterparts to solve their input characteristics using space-domain integral equation solutions.

Analysis of cylindrical wire dipole structures dates back to the 1930s when Hallen published the work on formulating the integral equation to solve the dipole antenna characteristics in [21]. Later researches [22-24] further extended the analysis of cylindrical antenna structures to correctly predict the current and impedance of such radiators. The article in [23] compares the solutions of Hallen's integral equation [21] for the cylindrical antenna given by Hallen, Bowkamp [25], Gray [26], King and Middleton [22], along with the transmission line theory of Schelkunoff [27] with experimental results for validation. From the discussions in [23], [24], it could be inferred that the method proposed by King and Middleton was more complex and demanded laborious mathematical exercise. In this condition, the induced EMF method [28] or Schelkunoff's method using a biconical antenna equivalent [28] can be proposed as easier and more comprehensible options in terms of an engineering solution for the problem. The authors reported an analysis of a CPW-fed strip monopole radiator using the induced EMF method, validated by simulations, which was published in [29]. Later, when it came to the analysis of equivalent slot structures it was found that the induced EMF method is suitable for dipole radiators whose electrical lengths lie between the ranges of $0.2 \lambda$ to $0.7 \lambda$ ( $\lambda$ being the wavelength corresponding to the frequency of operation) only [30]. Since the slots radiate efficiently at an electrical length of $\lambda$ (for their strip dipole equivalents) it leaves one solely with the method proposed by Schelkunoff, which uses a biconical antenna equivalent, for a simple yet convincing closedform analysis for the input characteristics of planar slot antennas on dielectric substrates. The derived expressions consist entirely of Sine and Cosine integral terms which are evaluated as convergent series summations [24] - contrary to complex numerical integrations, required in other methods of analysis which facilitates faster computation.

This work presents an analysis of CPW-fed slot antennas which are used to achieve a dual-polarized system with the help of Schelkunoff's Biconical Antenna Method [28]. However the classical approach of Schelkunoff's analysis is not sufficient to address the CPW-fed slot dipole structure as the latter includes a feed-point displacement in its configuration. It is to be noted that for wire dipole antennas the effect of feed-gap is reported [30], [31] and it is shown to influence the impedance and bandwidth characteristics of the structures. For CPW-fed strip monopole antennas, the gap between the bottom-most edge of the radiator and the top-most edge of the CPW grounds can be taken as an equivalent feed-gap parameter which is also reported [32] to have an impact on the impedance charac- teristics of the antennas. Therefore, for the analysis of $\mathrm{CPW}$-fed slot antennas, the feed-gap parameter is incorporated in the investigations presented in this article. The polarization purity of single-slot structures motivated the work to be extended towards a dual-polarized configuration in line with the works reported in [9], [10]. The proposed expressions for a CPW-fed single slot structure are further validated for the dual-polarized configuration in terms of the scattering parameters. In Sec. 2, the mathematical background of this analysis is presented. It is followed by the theoretical predictions validated by simulations and measurements of the proposed structures in Sec. 3 with the concluding remarks in Sec. 4 and the references.

\section{Problem Formulation}

Schelkunoff described the infinite biconical antenna as analogous to an infinite transmission line supporting a spherical wave for propagation [33]. The characteristic impedance of such a transmission line is formulated as,

$$
Z_{0}=120 \ln \left(\cot \frac{\theta_{\mathrm{hc}}}{2}\right)
$$

where $\theta_{\mathrm{hc}}$ is the half angle of the cone forming the biconical structure.

Figure 1(a) describes a finite biconical antenna with its geometric parameters. This finite structure can be modeled as a transmission line with a characteristic impedance of $Z_{0}$ terminated with a load as described in Fig. 1(b). Then at a distance $r$ from the source, the voltage and current relations on it is expressed as,

$$
\begin{aligned}
& V_{0}(r)=V_{0}(H) \cos [\beta(H-r)]+\mathrm{j} Z_{0} I_{0}(H) \sin [\beta(H-r)], \\
& I_{0}(r)=I_{0}(H) \cos [\beta(H-r)]+\mathrm{j} \frac{V_{0}(H)}{Z_{0}} \sin [\beta(H-r)] .
\end{aligned}
$$

Now, considering the feed-gap of the antenna to be $2 \delta$, as shown in Fig. 1(a) the input impedance of the structure is expressed as,

$$
\begin{aligned}
Z_{i} & =\frac{V_{0}(\delta)}{I_{0}(\delta)} \\
& =Z_{0} \frac{V_{0}(H) \cos [\beta(H-\delta)]+\mathrm{j} Z_{0} I_{0}(H) \sin [\beta(H-\delta)]}{Z_{0} I_{0}(H) \cos [\beta(H-\delta)]+\mathrm{j} V_{0}(H) \sin [\beta(H-\delta)]} .
\end{aligned}
$$

The equivalent terminal impedance $Z_{\mathrm{t}}$ being expressed as

$$
Z_{\mathrm{t}}=\frac{V_{0}(H)}{I_{0}(H)}=\frac{V(H)}{I(H)-\tilde{I}(H)} .
$$

$V_{0}$ and $I_{0}$ are the TEM mode voltage and current respectively. $I$ and $\tilde{I}$ represent total current and current due to the higher order modes in the structure [28]. The current $I(H)$ is the total current on the antenna at $r=H$ and therefore is the current flowing through the closing caps of the biconical 


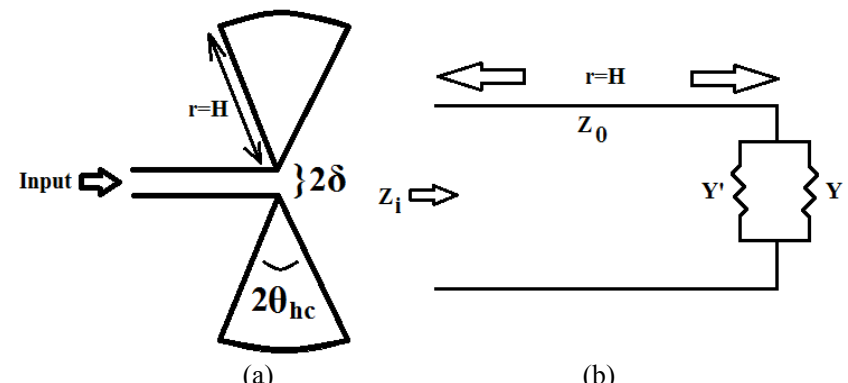

Fig. 1. (a) A finite biconical radiator and (b) its equivalent transmission line representation according to Schelkunoff's Analysis [28], [33].

structure. $I(H) / V(H)$ thus represents the admittance due to the capacitance between the closing caps. For thin radiators, the value of this capacitance is small and negligible [34]. Therefore, from (3) the input impedance $Z_{i}$ is expressed as,

$$
Z_{\mathrm{i}}=Z_{0} \frac{Z_{\mathrm{t}} \cos [\beta(H-\delta)]+\mathrm{j} Z_{0} \sin [\beta(H-\delta)]}{Z_{0} \cos [\beta(H-\delta)]+\mathrm{j} Z_{\mathrm{t}} \sin [\beta(H-\delta)]} .
$$

After calculations and simplifications, the expression for the flowing current due to the higher order modes is derived as [28],

$$
\begin{aligned}
& \tilde{I}(r)=-60 \frac{V_{0}(H)}{Z_{0}^{2}} \\
& \sum_{m=0}^{\infty} \frac{4 m+3}{(m+1)(2 m+1)}\left[\tilde{J}_{2 m+1}(\beta H)-\mathrm{j} \tilde{N}_{2 m+1}(\beta H)\right] \tilde{J}_{2 m+1}(\beta r),
\end{aligned}
$$

$\tilde{J}$ and $\tilde{N}$ being the Bessel's function coefficients of the first and second kind, respectively. The terminal admittance is then expressed as,

$$
Y_{\mathrm{t}}=-\frac{\tilde{I}(H)}{V(H)}=\frac{Z_{\mathrm{a}}(\beta H)}{Z_{0}^{2}}=\frac{R_{\mathrm{a}}(\beta H)+\mathrm{j} X_{\mathrm{a}}(\beta H)}{Z_{0}^{2}}
$$

where

$$
\begin{aligned}
& R_{\mathrm{a}}(\beta H)=60 \sum_{m=0}^{\infty} \frac{4 m+3}{(m+1)(2 m+1)} \tilde{J}_{2 m+1}^{2}(\beta H), \\
& X_{\mathrm{a}}(\beta H)=-60 \sum_{m=0}^{\infty} \frac{4 m+3}{(m+1)(2 m+1)} \tilde{J}_{2 m+1}(\beta H) \tilde{N}_{2 m+1}(\beta H) .
\end{aligned}
$$

Using (5) and (7), the input impedance of the antennas is expressed in terms of $Z_{\mathrm{a}}$ as,

\begin{tabular}{|c|c|c|c|}
\hline SI.No. & Method & $\begin{array}{l}\operatorname{Re} Z_{\text {IN }} \text { (in Ohms) for full } \\
\text { wave dipole with } \Omega=10\end{array}$ & \%error \\
\hline 1. & \begin{tabular}{l}
\multicolumn{3}{l}{ Experimental } \\
data from \\
Ph.D. Thesis of \\
D. D. King [23]
\end{tabular} & 800 & 0 \\
\hline 2. & $\begin{array}{l}\text { King } \\
\text { Middleton } \\
\text { second order } \\
\text { theory [23] } \\
\end{array}$ & 860 & 7.5 \\
\hline 3. & $\begin{array}{l}\text { Modified Gray } \\
\text { second order } \\
\text { theory [23], [24] }\end{array}$ & 885 & 13.5 \\
\hline 4. & $\begin{array}{l}\text { Hallen- } \\
\text { Bouwkamp } \\
\text { second order } \\
\text { theory [23], [25] }\end{array}$ & 1150 & 47.5 \\
\hline 5. & $\begin{array}{l}\text { Unmodified } \\
\text { Gray } 1.5 \text { order } \\
\text { theory [23], [26] }\end{array}$ & 740 & 7.5 \\
\hline 6. & $\begin{array}{l}\text { Schelkunoff } \\
\text { transmission } \\
\text { line theory (as } \\
\text { formulated } \\
\text { herein) [23], [27] }\end{array}$ & 740 & 7.5 \\
\hline
\end{tabular}

$$
Z_{\mathrm{i}}=Z_{0} \frac{Z_{\mathrm{a}} \sin [\beta(H-\delta)]-\mathrm{j} Z_{0} \cos [\beta(H-\delta)]}{Z_{0} \sin [\beta(H-\delta)]-\mathrm{j} Z_{\mathrm{a}} \cos [\beta(H-\delta)]} .
$$

For thin antennas $\left(\theta_{\mathrm{hc}} \rightarrow 0\right)$, Schelkunoff derived the expressions for (8) as,

$$
\begin{aligned}
& R_{\mathrm{a}}(\beta H)=60(\gamma+\ln (2 \beta H)-\mathrm{Ci}(2 \beta H))+ \\
& 30(\gamma+\ln (\beta H)-2 \mathrm{Ci}(2 \beta H)+\mathrm{Ci}(4 \beta H)) \cos (2 \beta H)+ \\
& 30(\operatorname{Si}(4 \beta H)-2 \operatorname{Si}(2 \beta H)) \sin (2 \beta H)
\end{aligned}
$$

Tab. 1. A comparison between the available methods [23] *[where $\Omega=2 \ln (2 h / a), h=$ half-length of the dipole, $a=$ radius of the dipole antenna].

$$
\text { and } \quad \begin{array}{ll}
X_{\mathrm{a}}(\beta H)=60 \operatorname{Si}(2 \beta H)+ \\
\\
30(\mathrm{Ci}(4 \beta H)-\ln (\beta H)-\gamma) \sin (2 \beta H)- \\
\\
30 \mathrm{Si}(4 \beta H) \cos (2 \beta H))
\end{array}
$$

where $\gamma$ is the Euler's constant.

In order to further justify the use of Schelkunoff's analytical technique, a comparison of the available methods based on [23] is also given in Tab. 1. It may be noted that the methods 2, 3, 4 and 5 are based on Hallen's Integral equation. The solution methods are iterative in nature and therefore they call for large computation resources. On the other hand Schelkunoff's transmission line theory utilizes direct computation involving Cosine and Sine integrals. The comparison shows that the corresponding percentage error is also reasonably small so as to meet the purpose of the designer.

\section{Antenna Geometry and Design}

Figure 2(a) describes a CPW-fed slot dipole antenna which can be taken as the complementary structure of a strip-dipole radiator [35] (Fig. 2(b)). For dipole antennas having non-circular cross-sections, Hallen [21] proposed an equivalent radius model which maps a slot of width $w$ to an equivalent dipole structure having a radius $a$,

$$
a=0.25 w .
$$

Table 2 depicts the dimensions of the prototype structure. Since the value of $a$ suggests a thick dipole structure, Schelkunoff's Biconical Antenna Model is used to analyze the equivalent problem and thus an equivalent 


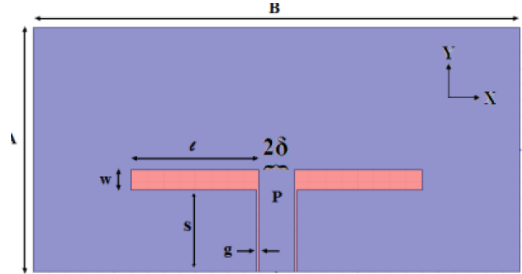

(a)

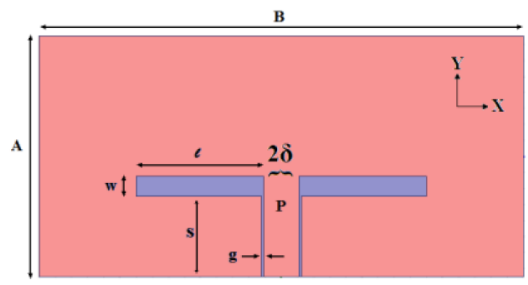

(b)

Fig. 2. (a) Proposed CPW-fed slot dipole antenna and (b) its strip equivalent [35].

\begin{tabular}{|c|c|}
\hline Name of the Parameter & Value (in mm) \\
\hline $\boldsymbol{A}$ & 30 \\
\hline $\boldsymbol{B}$ & 60 \\
\hline$\ell$ & $(18-\delta)$ \\
\hline $\boldsymbol{w}$ & 2.5 \\
\hline $\boldsymbol{g}$ & 0.3 \\
\hline $\boldsymbol{2} \boldsymbol{\delta}$ & 4.46 \\
\hline $\boldsymbol{s}$ & 10 \\
\hline
\end{tabular}

Tab. 2. Dimensions of the prototype structure.

biconical structure is considered having appropriate values for $\theta_{\mathrm{hc}}$ and $H$ as follows,

$$
\theta_{\mathrm{hc}}=\tan ^{-1}\left(\frac{a}{\ell}\right) \text { and } H=\ell \text {, for small values of } \theta_{\mathrm{hc}}
$$

While carrying out the analysis for antennas on dielectric substrates, the expression for characteristic impedance of the infinite biconical antenna equivalent is augmented as

$$
Z_{0}=\frac{120}{\sqrt{\varepsilon_{\text {eff }}}} \ln \left(\cot \frac{\theta_{\mathrm{hc}}}{2}\right)
$$

where $\varepsilon_{\text {eff }}$ is the effective dielectric constant for the proposed structure in Fig. 2(a). The propagation constant $\beta$ along with wavelength $\lambda$ are also accordingly modified in the equations numbering (9-13) for the calculations. Equations (9-14) are used to predict $Z_{\mathrm{i}}$ at the point $\mathrm{P}$ (can be viewed as the exact feed-point of the radiator, accounting the feed-gap) shown in Fig. 2(b) for the equivalent strip-dipole structure. To map the impedance at $\mathrm{P}$ on to the proposed slot structure shown in Fig. 2(a), $Z_{\mathrm{i}}$ is modified using the Babinet's principle [14] as

$$
Z_{\mathrm{i}_{\text {slot }}}=\frac{\eta^{2}}{4 Z_{\mathrm{i}}} \text { where } \eta=120 \pi \text {. }
$$

In this expression far field condition is assumed that ensures that the fields are evaluated in free space. Also, in reference [35] the authors too have used free space value of intrinsic impedance to derive their results and expressions under a similar situation. By assuming the characteristic impedance of the CPW-feed to be $50 \Omega$, the value of $S_{11}$ on the line is calculated as,

$$
S_{11}=20 \log _{10}\left|\frac{Z_{\mathrm{i}_{\text {slot }}}-50}{Z_{\mathrm{i}_{\text {slot }}}+50}\right| .
$$

Now, the relevant algorithm steps for the C-program which is used to calculate the values of $S_{11}$ are as follows,

- Read antenna data as per the geometric dimensions provided.

- Compute $R_{\mathrm{a}}$ and $X_{\mathrm{a}}$ using (10) and (11).

- Compute $Z_{0}$ using (13) and (14).

- Compute $Z_{\mathrm{t}}$ using (9).

- Compute $Z_{\text {slot }}$ using (15).

- Compute $S_{11}$ using (16).

\section{Results and Discussions}

The simulations for the proposed structure is carried out in ANSYS HFSS [36] and the simulated results of $S_{11}$, for different values of the width $w$ of the slot, are compared with the theoretical predictions as shown in Fig. 3. As in the figure, the change in $w$ mostly affects the matching characteristics of the structure and the changes are wellreflected in the theoretical predictions.

A prototype is fabricated (shown in Fig. 4(a)) as per the dimensions mentioned in Tab. 1, on an FR-4 Epoxy substrate $\left(\varepsilon_{\mathrm{r}}=4.3\right.$, substrate thickness $\left.0.732 \mathrm{~mm}\right)$ and the theoretical results are compared with the simulations and the measured outputs in Fig. 4(b).

From Tab. 2 of the article [23], it is evident that the theory of Schelkunoff becomes less accurate at lower electrical lengths corresponding to lower frequencies. Therefore at the lower frequency points of the input reflection coefficient plots, slight mismatches are expected, which have led to the mismatches in bandwidth predictions. Moreover, at the higher frequencies, including at and around the frequency of resonance, the plots are almost identical in the figures concerned.

Simulated and measured radiation patterns on $\mathrm{XZ}$ and YZ planes (refer to Fig. 2) are given in Fig. 5(a-b) which resembles the dipole characteristics. The measured gain of the structure is $5.25 \mathrm{~dB}$ which qualifies itself as a practical radiator suitable for regular applications. Thus the theory formulated for such a CPW-fed slot radiator is validated, and the structure is also found to satisfy the basic features of the configuration. The slightly directive pattern observed in Fig. 5(b) could be attributed to the finite size of the substrate according to the authors.

To extend the design for dual-polarized applications, orthogonally placed radiators are utilized as in [9], [10]. 


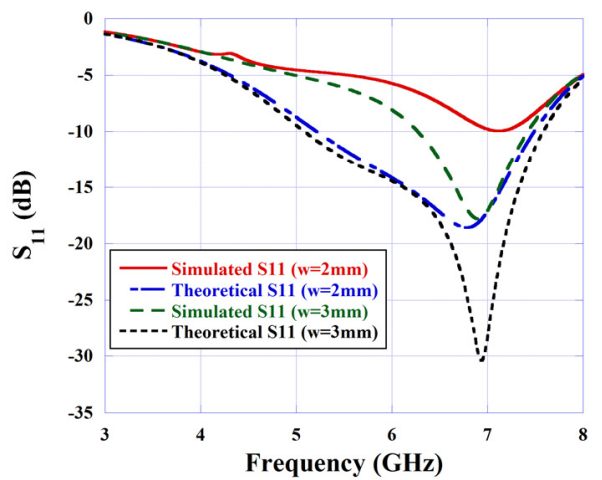

Fig. 3. Simulated and predicted plots of $S_{11}$ for different values of $w$.

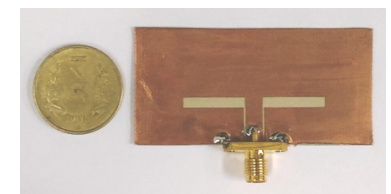

(a)

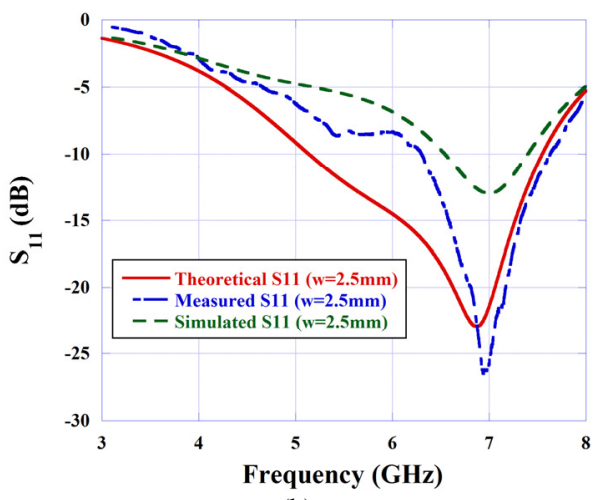

(b)

Fig. 4. (a) The fabricated prototype and (b) the comparison of the simulated, theoretical and measured $S_{11}$ characteristics.

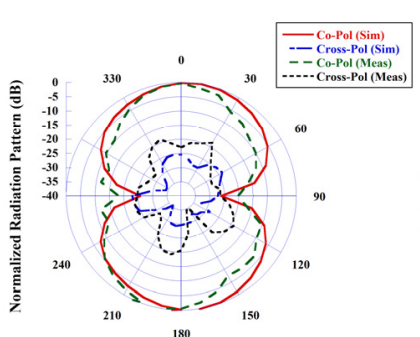

(a)

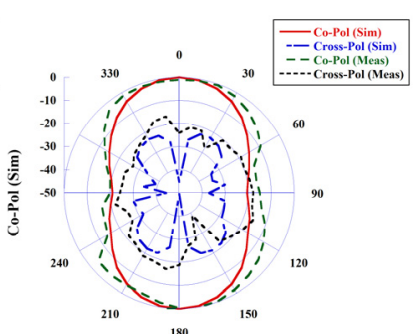

(b)
Fig. 5. Simulated and measured radiation patterns for the CPW-fed slot dipole structure on the (a) XZ-plane and the (b) YZ-plane respectively, at $6.9 \mathrm{GHz}$.

Figure 6 depicts the configuration, which is developed to facilitate dual-polarized radiation. It is predicted that orthogonally placed slot dipoles, owing to their inherent polarization purity, would be able to yield dual-polarized radiation when individual ports, shown in Fig. 6, are excited. The new dimensions are: $A=B=55 \mathrm{~mm}, \ell_{2}=$ $26.5 \mathrm{~mm}, \ell_{3}=24.97 \mathrm{~mm}$. Figure 7 represents the simulated surface current distributions on the slot dipoles at $6.9 \mathrm{GHz}$ when each port is excited at a time with the other terminated by a matched load. The distributions show little coupling between the radiating elements suggesting high isolation between the ports which is a necessity to establish the dual-polarized characteristics. From the distributions, it is evident that for each port excitation, the surface currents are mostly concentrated on the individual dipole slots only. This observation establishes the characteristic dipole-like radiation obtained from the individual elements. When port 1 is excited, signal from the lower arm of the vertical slot gets slightly coupled to the horizontal slot connected to port 2 which is visible from the noticeable current density at the edges of the horizontal slot. However the two slots being orthogonal such (reactive) coupling does not produce any significant power output at port 2 and therefore does not contribute to $S_{21}$ which is evident from Fig. 9(b). This fact was also checked by simulation studies prior to the measurements and it ensured that the values of $S_{21}$ and $S_{12}$, signifying isolation lie below $-25 \mathrm{~dB}$ for the entire frequency range of interest. Also, the surface current is almost negligible on the other parts of the substrate which shows that the dimensions of it have little or no effect on the radiator performance. This is also the reason that, even though the proposed theory, models the individual dipole slots without considering the finite substrate dimensions - it succeeds to predict the resonance characteristics sufficiently well. The orthogonal surface current concentrations around each dipole slots for each port excitation, justifies the claim of dual-polarized radiation.

The theoretical expressions (9-16) are used to predict the individual input reflection coefficient characteristics at both the ports again. The compared results of $S_{11}$ and $S_{22}$ among the simulations, measurements and the theoretical predictions are provided in Figs. 8(a,b). The prototype of

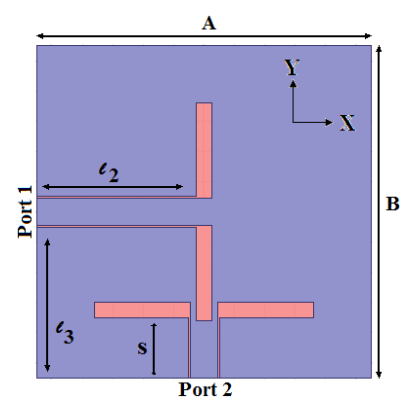

Fig. 6. Proposed CPW-fed dual-polarized slot dipole structure.

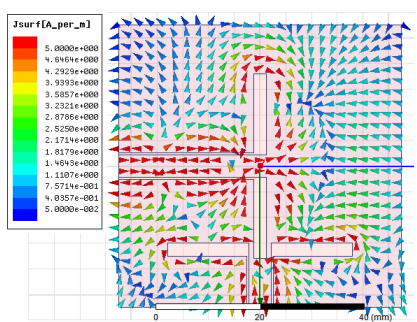

(a)

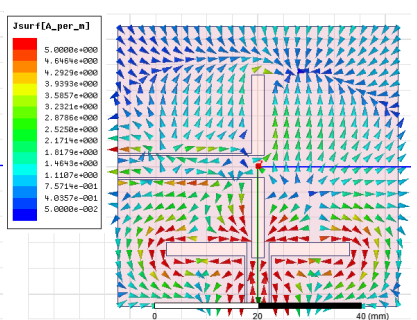

(b)
Fig. 7. Surface current distributions (a) when port 1 is excited and (b) when port 2 is excited at $6.9 \mathrm{GHz}$. 
the proposed structure is shown in Fig. 9(a) and the measured S-parameters are depicted in Fig. 9(b). Since, the isolation between the ports are good - as depicted by the measured $S_{21}$ and $S_{12}$ plots - mutual impedance between the structures are not accounted for while using the expressions. In spite of this - the measured results for $S_{11}$ and $S_{22}$ matched sufficiently well with the theoretical predictions, as depicted in Fig. 8.

The formation of small ripples in Fig. 8(a) is probably due to the measurement inaccuracies and cable issues which also created jitters in the curve depicted in Fig. 8(b). However, even at the presence of ripples the measured curve follows the pattern of the theoretically predicted results. This validates the suitability of the proposed theory for the analyses. In Fig. 9(b) the responses of $S_{21}$ and $S_{12}$ are identical which validates the reciprocal nature of the configuration.

Theoretical understanding of the present structure(s) requires analysis of dipole antenna of length $\lambda$ with a considerably large displacement of feed points along the dipole axis. It may also be noted that the input behavior of the dipole depends mostly on the reactance characteristics and the accuracy of such result greatly depends on the modeling of the input region [30], [37]. The presence of gap makes the problem more intricate. In the work, the authors have modified Schelkunoff's method to include the gap problem. This is necessary to estimate both the resonance frequency and the bandwidth behavior. To critically evaluate the performance of the theory it is better to consider the single slot case. From Fig. 4 it may be noted that the expressions could predict the theoretical behavior very close

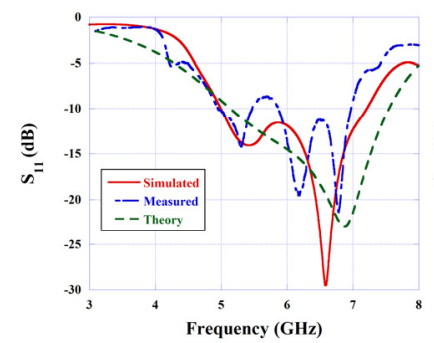

(a)

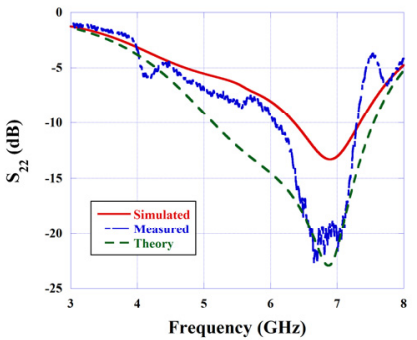

(b)
Fig. 8. The simulated, measured and theoretical plots of (a) $S_{11}$ and (b) $S_{22}$ respectively.

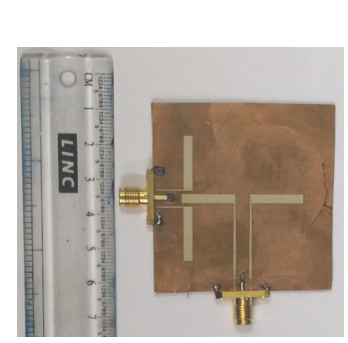

(a)

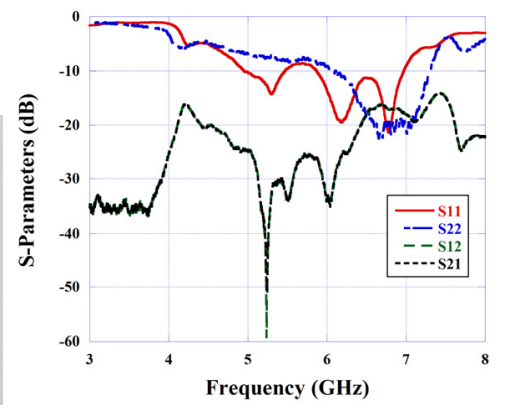

(b)
Fig. 9. (a) The fabricated prototype and (b) the measured $\mathrm{S}$-parameters of the structure.

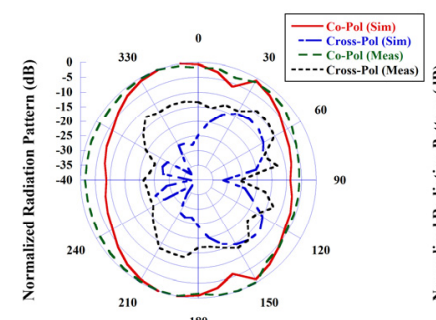

(a)

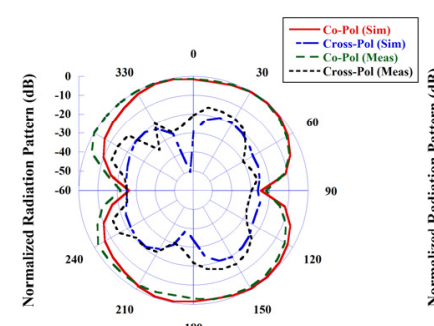

(c)

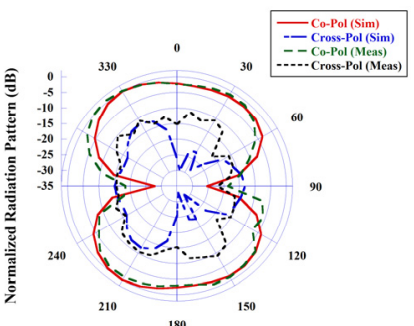

(b)

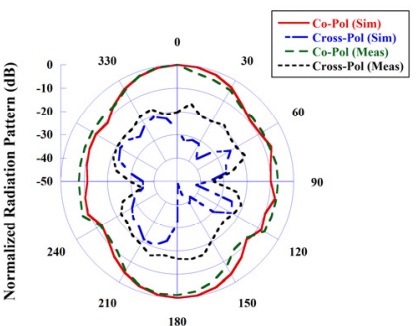

(d)
Fig. 10. Simulated and measured radiation patterns for (a) Port 1, XZ plane, (b) Port 1, YZ plane, (c) Port 2, XZ plane, (d) Port 2, YZ plane; at $6.9 \mathrm{GHz}$.

to the resonance frequency quite well, but there are discrepancies at lower frequencies. However at higher frequencies the agreement again is good. Similar nature may be observed in [20], where the agreement of the theoretical analysis with the experimental result is slightly better because the feed width of the CPW line is extremely small compared to the wavelength. Simulated and radiation patterns of the dual-polarized structure are depicted in Fig. 10(a-d) for the respective planes. The patterns follow their typical characteristics of dipole structures and confirm the fact of orthogonal radiation to validate dual-polarized performance.

The measured antenna gain at port 1 and 2 are $3.67 \mathrm{~dB}$ and $3.54 \mathrm{~dB}$, respectively. The slight discrepancies observed in the measured patterns can be accounted to the fabrication inaccuracies or the limitations of the practical measurement setups at the laboratories. On an average, in the broadside direction the structures provide acceptable cross-pol performances over an optimum angular range as depicted in the figures. Figure 11 depicts the gain vs. frequency curves for the proposed structures (for the single

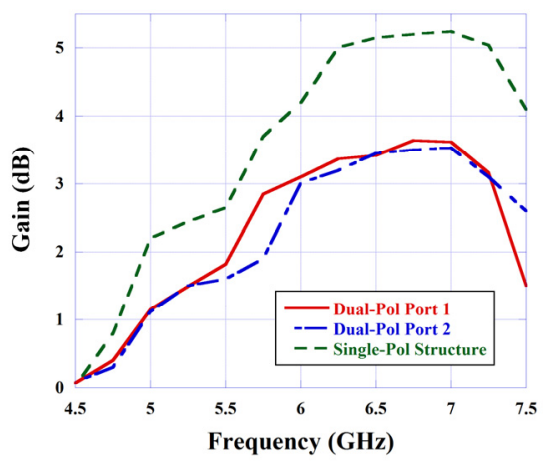

Fig. 11. Measured gain vs. frequency curves for the single element as shown in Fig. 2, and for both the elements of the dual-polarized configuration as in Fig. 6. 
element as shown in Fig. 2, and for both the elements of the dual-polarized configuration, as in Fig. 6). The curves represent stable gain characteristics over the measured impedance bandwidths depicted in Figs. 4 and 8.

As was noted in Sec. 2 of this article, most of the numerical/computational solution methods available for solving the Hallen's integral equation are iterative in nature. Therefore, they call for the usage of more computational resources as well as computational time. The proposed method of using Schelkunoff's transmission line theory for the problem offers closed form sine and cosine integral based expressions. This method therefore is efficient both in terms of computational time and resources. The expressions were evaluated using TURBO C-based program codes developed by the authors and the computational requirements are compared with that of the HFSS platform, the latter being an FEM-based simulation environment. Since, the objective of this article was primarily to predict the input characteristics of the proposed structures, during the computational resource comparison - the HFSS program was simulated without calculating the farfields radiations - so it may be tallied with the TURBO Cprogram which returns the S-parameter results. The Cprogram returns the input characteristics of the proposed slot(s) as an individual element(s). HFSS uses comparable amount of CPU memory for both the single and dual-polarized structures, however the time for computation changes for the latter. The results are depicted in Tab. 3 for the readers.

\begin{tabular}{|c|c|c|c|}
\hline $\begin{array}{l}\text { Sl. } \\
\text { No. }\end{array}$ & $\begin{array}{l}\text { Simulation } \\
\text { Method }\end{array}$ & $\begin{array}{l}\text { TURBO C-based } \\
\text { Closed Form } \\
\text { Analytical Solution }\end{array}$ & $\begin{array}{c}\text { FEM-Based } \\
\text { Simulation (HFSS) }\end{array}$ \\
\hline \multirow{2}{*}{1.} & \multirow{2}{*}{$\begin{array}{l}\text { CPU Time } \\
\text { Taken (in } \\
\text { Seconds) }\end{array}$} & \multirow{2}{*}{1} & $\begin{array}{c}32 \text { (Single-Polarized } \\
\text { Element) }\end{array}$ \\
\hline & & & $\begin{array}{c}168 \text { (Dual-Polarized } \\
\text { Element) }\end{array}$ \\
\hline \multirow{2}{*}{2.} & $\begin{array}{c}\text { CPU } \\
\text { Memory }\end{array}$ & \multirow{2}{*}{$* 5$} & $\begin{array}{c}5 \text { (Single-Polarized } \\
\text { Element) }\end{array}$ \\
\hline & $\begin{array}{l}\text { Consumed } \\
\text { (in MB) }\end{array}$ & & $\begin{array}{c}75.6 \text { (Dual-Polarized } \\
\text { Element) }\end{array}$ \\
\hline
\end{tabular}

Tab. 3. Computational resource comparison for the proposed method against an FEM-based simulator.

[Computer Specification: Intel(R) Core(TM)2 Duo CPU E7200 (a) $2.53 \mathrm{GHz} 2.53 \mathrm{GHz}$, Installed RAM: $3.00 \mathrm{~GB}$, single core operation for each cases]

*The TURBO C compiler takes $5 \mathrm{MB}$ of CPU Memory to function on the Windows Platform. The authors were unable to find the exact CPU memory, consumed by the individual program. However, under all circumstances it will be less than or equal to $5 \mathrm{MB}$ which justifies this comparison.

\section{Conclusions}

Analysis of CPW-fed slotted dipole structure is reported with the help of Schelkunoff's biconical antenna analysis technique to investigate the input characteristics of such configurations. The polarization purity of single-slot structures motivated the work to be extended towards a dual-polarized configuration. The developed theory is further validated for the same and the measured results tallied with the predictions at both the ports. The surface current distributions of the dual-polarized structure are provided to justify the claim of isolation. Radiation patterns and gain measurements are carried out for both the configurations to register their effective radiating performances. Application areas of such structures are wide and encouraging in the fields of communication, home appliances, and other microwave components.

\section{Acknowledgments}

This work is supported by CSIR, Govt. of India (ack. no.: 143126/2K17/1 and File No. 09/096(0925)/2018EMR-I, dated: 26/04/2018). The authors would also like to thank the anonymous reviewers for their valuable comments and advices on this article which has improved the document in adequate measures.

\section{References}

[1] LEE, W. C. Y., YEH, Y. S. Polarization diversity system for mobile radio. IEEE Transactions on Communication, 1972, vol. 20, no. 5, p. 912-923. DOI: 10.1109/TCOM.1972.1091263

[2] TU, W. H., CHANG, K. Miniaturized CPW-fed slot antenna using stepped impedance resonator. In Proceedings of IEEE Antennas Propagation Society International Symposium. Washington DC (USA), 2005, p. 351-354. DOI: 10.1109/APS.2005.1552663

[3] TU, W. H. Compact harmonic-suppressed coplanar waveguide-fed inductively coupled slot antenna. IEEE Antennas and Wireless Propagation Letters, 2008, vol. 7, p. 543-545. DOI: 10.1109/LAWP.2008.2002905

[4] CHEN, Y. C., CHEN, S. Y., HSU, P. A modified CPW-fed slot loop antenna with reduced cross polarization and size. IEEE Antennas and Wireless Propagation Letters, 2011, vol. 10, p. 1124-1126. DOI: 10.1109/LAWP.2011.2171471

[5] WU, C. M. Dual-band CPW-fed cross-slot monopole antenna for WLAN operation. IET Microwaves, Antennas and Propagation, 2007, vol. 1, no. 2, p. 542-546. DOI: 10.1049/iet-map:20050116

[6] CHEN, S. Y., HSU, P. Broad-band radial slot antenna fed by coplanar waveguide for dual-frequency operation. IEEE Transactions on Antennas and Propagation, 2005, vol. 53, no. 11, p. 3448-3452. DOI: 10.1109/TAP.2005.858574

[7] DING, X., JACOB, A. F. CPW-fed slot antenna with wide radiating apertures. IEE Proceedings - Microwaves, Antennas and Propagation, 1998, vol. 145, no. 1, p. 104-108. DOI: 10.1049/ipmap:19981629

[8] CHIU, C. Y., YAN, J. B., MURCH, R. D., et al. Design and implementation of a compact 6-port antenna. IEEE Antennas and Wireless Propagation Letters, 2009, vol. 8, p. 767-770. DOI: 10.1109/LAWP.2009.2026663

[9] LI, W., ZENG, Z., YOU, B., et al. Compact dual polarized printed slot antenna. IEEE Antennas and Wireless Propagation Letters, 2017, vol. 16, p. 2816-2819. DOI: 10.1109/LAWP.2017.2748542

[10] HETTAK, K., DELISLE, G. Y., STUBBS, M. G. A novel variant of dual polarized CPW fed patch antenna for broadband wireless communications. In Proceedings of IEEE Antennas Propagation 
Society International Symposium. Salt Lake City (USA), 2000, p. 286-289. DOI: 10.1109/APS.2000.873765

[11] KOLUNDZIJA, B. M., OGNJANOVIC, J. S., SARKAR, T. K. WIPLD: Electromagnetic Modeling of Composite Metallic and Dielectric Structures. Norwood (MA): Artech House, 2000. ISBN: 978-0890063583

[12] TAFLOVE, A., UMASHANKAR, K. R. The finite-difference time-domain method for numerical modeling of electromagnetic wave interactions. Electromagnetics, 1990, vol. 10, no. 1, p. 105-126. DOI: $10.1080 / 02726349008908231$

[13] JONES, D. S. Methods in Electromagnetic Wave Propagation. Oxford (UK): Clarendon Press, 1979

[14] BOOKER, H. G. Slot aerials and their relation to complementary wire aerials. Journal of the IEE, Part IIIA: Radiolocation, 1946, vol. 93, no. 4, p. 620-626. DOI: 10.1049/ji-3a-1.1946.0150

[15] KOMINAMI, M., POZAR, D. M., SCHAUBERT, D. H. Dipole and slot elements and array on semi-infinite substrate. IEEE Transactions on Antennas and Propagation, 1985, vol. 33, no. 6, p. 600-607. DOI: 10.1109/TAP.1985.1143638

[16] TEKIN, I., NEWMAN, E. H. Space-domain method of moments solution for a strip on a dielectric slab. IEEE Transactions on Antennas and Propagation, 1998, vol. 46, no. 9, p. 1346-1348. DOI: $10.1109 / 8.719978$

[17] POPOVIC, B. D., NESIC, A. Generalisation of the concept of equivalent radius of thin cylindrical antennas. IEE Proceedings $H$ Microwaves, Optics and Antennas, 1984, vol. 131, no. 3, p. 153-158. DOI: 10.1049/ip-h-1.1984.0033

[18] MOORE, J., WEST, M. A. Simplified analysis of coated wire antennas and scatterers. IEE Proceedings on Microwaves, Antennas and Propagation, 1995, vol. 142, no. 1, p. 14-18. DOI: 10.1049/ip-map:19951651

[19] LAOHAPENSAENG, C., FREE, C. Simplified integral equation for analyzing the printed strip dipole antenna. IEE Proceedings on Microwaves, Antennas and Propagation, 2006, vol. 153, no. 3, p. 301-306. DOI: 10.1049/ip-map:20050022

[20] NESIC, A., POPOVIC, B. D. Analysis of slot antenna on dielectric substrate. IEE Proceedings H, Microwaves, Optics and Antennas, 1985, vol. 132, no. 7, p. 474-476. DOI: 10.1049/ip-h-2.1985.0085

[21] HALLEN, E. Theoretical investigations into the transmitting and receiving qualities of antennae. Nova Acta Regiae Societatis Scientiarum Upsaliensis, ser. IV, vol. 11, no. 4, 1938, p. 1-44.

[22] KING, R., MIDDLETON, D. The cylindrical antenna; Current and impedance. Quarterly of Applied Mathematics, 1946, vol. 3, no. 4. DOI: $10.1090 / \mathrm{qam} / 15323$

[23] MIDDLETON, D., KING, R. The thin cylindrical antenna: A comparison of theories. Journal of Applied Physics, 1946, vol. 17, no. 4, p. 273-284. DOI: 10.1063/1.1707714

[24] KING, R. W. P. The Theory of Linear Antennas. Harvard University Press, Cambridge Massachusetts, 1956

[25] BOUWKAMP, C. J. Note on an Integral Occurring in Antenna Theory. Natuurkundig Laboratorium de N. V. Philips' Gloeilampenfabrieken, Eindhoven, Netherlands. Unpublished

[26] GRAY, M. C. A Modification of Hallén's solution of the antenna problem. Journal of Applied Physics, 1944, vol. 15, no. 1, p. 61-65. DOI: $10.1063 / 1.1707368$

[27] SCHELKUNOFF, S. A. The electromagnetic theory of coaxial transmission lines and cylindrical shields. The Bell System Technical Journal, 1934, vol. 13, no. 4, p. 532-579. DOI: 10.1002/j.1538-7305.1934.tb00679.x

[28] JORDAN, E. C., BALMAIN, K. G. Electromagnetic Waves and Radiating Systems. Ch. 14. Prentice-Hall of India (Pvt.), 1964
[29] BANERJEE, A., BANDYOPADHYAY, A. K. Theoretical investigation on the input impedance of a CPW-fed strip monopole antenna. Microwave and Optical Technology Letters, 2017, vol. 59, no. 2, p. 346-348. DOI: 10.1002/mop.30287

[30] ESTARKI, M. D., VAUGHAN, R. G. Theoretical methods for the impedance and bandwidth of the thin dipole. IEEE Antennas and Propagation Magazine, 2013, vol. 55, no. 1, p. 62-81. DOI: 10.1109/MAP.2013.6474485

[31] BANDYOPADHYAY, A. K., CHOWDHURY, S. K. Some investigations on the feed points displaced dipole. IETE Journal of Research (India), 1973, vol. 19, no. 12, p. 686-688. DOI: $10.1080 / 03772063.1973 .11487298$

[32] BANERJEE, A., CHATTERJEE, S., GUPTA, B., et al. Theoretical investigation on input characteristics of CPW-fed wide rectangular monopole structures. In IEEE International Conference on Antenna Innovations \&Modern Technologies for Ground, Aircraft and Satellite Applications (iAIM). Bangalore (India), 2017, p. 1-5. DOI: 10.1109/IAIM.2017.8402614

[33] KRAUSS, J. D. Antennas. $2^{\text {nd }}$ ed. Tata McGraw-Hill, 1997 (Ch. 8)

[34] JEANS, J. H. Electricity and Magnetism. London (UK): Cambridge Press, 1946. P. 249.

[35] NESIC, A. Slotted antenna array excited by a coplanar waveguide. Electronics Letters, 1982, vol. 18, no. 6, p. 275-276. DOI: 10.1049/el:19820188

[36] Ansoft Corp HFSS v.13

[37] COLLIN, R. E., ZUCKER, F. J. Antenna Theory. N.Y.: McGrawHill, 1969. Part 1, p. 346. ISBN: 0070117993

\section{About the Authors ...}

Amartya BANERJEE (corresponding author) received his M.Tech. from IIEST, Shibpur in 2016. His research interests include microwave design techniques and circuit theoretical analysis.

Kaushik PATRA received his M.E. from Jadavpur University, Kolkata in 2012. His research interests include approximate modeling for microstrip lines for EMC calculation and application in travelling wave and leaky wave antennas.

Sayan CHATTERJEE is currently an Associate Professor at the Dept. of Electronics and Telecommunication Engineering, Jadavpur University. His research interest includes microwave and millimeter wave antennas, passive devices and slotted array antenna

Bhaskar GUPTA is currently a Professor in the Dept. of ETCE, Jadavpur University. His present area of interest is planar antennas, dielectric resonator antennas, wearable antennas, computational electromagnetic, MEMS design and application of soft computing techniques in microwave engineering.

Anup Kumar BANDYOPADHYAY is a retired Professor from the Dept. of ETCE, Jadavpur University, Kolkata. His research interests include microwave and antenna engineering, circuit theory and program proving. 